\title{
The Association Between Pre-Pregnancy Body Mass Index, Perinatal Depression and Maternal Vitamin D Status: Findings from an Australian Cohort Study
}

\author{
Rati Jani (iD) \\ Catherine R Knight-Agarwal (D) ' \\ Michael Bloom (iD) ${ }^{2}$ \\ Monica Yuri Takito ${ }^{3}$ \\ 'The University of Canberra, School of \\ Clinical Sciences, Faculty of Health, \\ Canberra, ACT, Australia; ${ }^{2}$ Northern \\ Sydney Local Area Health District, \\ Sydney, NSW, Australia; ${ }^{3}$ School of \\ Physical Education and Sport, The \\ University of Sao Paulo, Sao Paulo, Brazil
}

This article was published in the following Dove Press journal: International Journal of Women's Health

Purpose: This study aimed to assess the relationship between early-pregnancy Body Mass Index (BMI), perinatal depression risk and maternal vitamin D status.

Patients and Methods: A retrospective cohort study from 2013 to 2017 was undertaken involving 16,528 birth events in the Australian Capital Territory. Multivariate binary logistic regression was conducted using the forced entry method. Mediation of the association between maternal early-pregnancy BMI and perinatal depression risk by vitamin D status was also tested. Results: Adjusted logistic regression models found that high maternal early-pregnancy BMI was associated with increased risk of developing perinatal depression (AOR 1.421; 95\% CI, $1.191,1.696$ ) as well as increased odds of being vitamin D deficient (AOR 1.950; 95\% CI; $1.735,2.191)$. In comparison to women with low perinatal depression risk, women with high perinatal depression risk had increased odds of being vitamin D deficient (AOR 1.321; 95\% CI, 1.105, 1.579). Maternal early-pregnancy BMI was a weak significant predictor of perinatal depression risk after including vitamin $\mathrm{D}$ as a mediator, consistent with partial mediation, Path $\mathrm{C}$ : $\mathrm{B}=0.016$ (95\% CI 1.003, 1.030), $\mathrm{p}=0.02$. Path $\mathrm{C}^{\prime}: \mathrm{B}=0.014(95 \% \mathrm{CI}$ $1.001,1.028), \mathrm{p}=0.04$.

Conclusion: In line with current Australian recommendations, women with high earlypregnancy BMI should be screened for both perinatal depression risk and vitamin D deficiency, with referral to relevant support services when indicated.

Keywords: overweight, obesity, pregnancy nutrition, depression

\section{Introduction}

Globally, in 2014 it was estimated that there were 38.9 million overweight pregnant women of which 14.6 million were obese. ${ }^{1}$ In Australia, there is an increasing incidence of obesity in young women with their National Health Survey reporting $42.4 \%$ of women in the peak child-bearing years of 25-34 to be overweight or obese. ${ }^{2}$ It is widely accepted that a high body mass index (BMI) during pregnancy increases the risk of various adverse obstetric outcomes including pregnancyinduced hypertension, caesarean section, macrosomia, depression and stillbirth. ${ }^{3-6}$ Maternal obesity is a notable risk factor for vitamin D deficiency which may be related to the sequestering of D3 into adipose tissue. ${ }^{7}$

Vitamin D acts as a unique neuro-steroid hormone which is required for normal brain development, homeostasis and function. Pregnancy is a time of susceptibility to Vitamin
Correspondence: Rati Jani

The University of Canberra, School of

Clinical Sciences, Faculty of Health,

Canberra, ACT, Australia

Tel +6I 407806663

Fax +6I 262015040

Email rati.jani@canberra.edu.au 
D deficiency, due to the fact that women must sustain their own vitamin D stores as well as those of their unborn child. ${ }^{7-10}$

On average $10-20 \%$ of women experience depression during pregnancy, and approximately 1 in 7 women experience depression within the first year after giving birth. ${ }^{11-15}$ In their systematic review, Aghajafari et $\mathrm{al}^{8}$ reported an inverse association between vitamin D and Edinburgh Postnatal Depression Scale (EPDS) scores during the antenatal period in 5 of 7 studies. While the results in their review varied, overall they indicate a significant association between maternal vitamin $\mathrm{D}$ status and both antenatal and postnatal depression. ${ }^{8}$

Given that pregnancy may be a time of vulnerability to vitamin D deficiency, which, in turn, may lead to a predisposition for depression we sought to examine the relationship between maternal serum vitamin $\mathrm{D}$ and the risk of perinatal depression in an obstetric cohort of Australian women with varying BMI.

\section{Methods}

\section{Study Design and Participants}

This retrospective cross-sectional, cohort study used data from the Birthing Outcomes System (BOS) at a major tertiary institution in the Australian Capital Territory (ACT) between the 1st of January 2013 and to the 31st of December 2017. In that time, there were 17,132 birth events with the hospital being the major maternity centre for a catchment population of 550,000. Women with missing BMI data and multiple pregnancies were excluded leaving 16,528 women for analysis. The ethics committee who approved this study did not require written consent as the researchers received the birthing outcomes secondary data de-identified.

\section{Maternal BMI}

Maternal BMI was derived from measured height and weight recorded at the first antenatal visit (usually at 12-14 weeks gestation). In BOS, BMI values are rounded up or down to the nearest whole number according to scientific notation. BMI was categorised into four groups; underweight $\left(\leq 18 \mathrm{~kg} / \mathrm{m}^{2}\right)$; normal weight $\left(19-24 \mathrm{~kg} / \mathrm{m}^{2}\right)$; overweight $\left(25-29 \mathrm{~kg} / \mathrm{m}^{2}\right)$; obese $\left(\geq 30 \mathrm{~kg} / \mathrm{m}^{2}\right) .{ }^{16}$

\section{Pregnancy-Related Depression Risk}

At the facility where this study was conducted, the Edinburgh Postnatal Depression Scale (EPDS) is used to screen depression risk in all women receiving routine antenatal care. The EPDS is a 10 -item self-rating scale, which was developed by Cox, Holden and Sagovsky. ${ }^{17}$ It has been validated as a reliable screening tool for assessing pregnancy-related depression. ${ }^{17}$ Each of the 10 answers is given a score of 0 to 3 with a maximum score is 30 . Every woman at the study hospital is invited to take this questionnaire at around 12-14 weeks gestation and are asked to select the answer which best describes how they have felt over the past 7 days. A high EPDS score is defined $\geq 13$ as originally validated by Cox, Holden and Sagovsky. ${ }^{17}$ Following the definition, EPDS scores were categorised for analysis as high risk $(\geq 13)$ and low to moderate risk $(<13)$.

\section{Vitamin D Status}

The Royal Australian and New Zealand College of Obstetricians and Gynaecologists (RANZCOG) recommends testing be considered for pregnant women at high risk of suboptimal vitamin D levels. ${ }^{16}$ Historically (2008-2012) the vitamin D status of pregnant women has not been routinely recorded in the BOS at the study hospital. However, from 2013 onwards, maternal vitamin D status was measured more routinely and has only been documented in BOS as a bicategorical variable (vitamin D deficient vs non-deficient). Blood samples are collected by the hospital's pathology department at around 14 weeks gestation with a serum vitamin $\mathrm{D}$ level of $\leq 50 \mathrm{nmol} / \mathrm{L}$ considered low according to published guidelines. ${ }^{16}$

\section{Data Analysis}

Descriptive analysis was reported using means and standard deviations or frequency and percentages for continuous and categorical variables, respectively. Bivariate and multivariate analysis between early-pregnancy BMI (underweight, healthy weight, overweight, obese), vitamin D status (deficient vs not deficient) and perinatal depression risk (high vs low risk) as categorical variables were examined using binary logistic regression. For multivariate binary logistic regression, covariates, namely, mother's country of birth, marital status, mother's working status, smoking, domestic violence, hypertension during pregnancy, season of birth, gender (neonate), maternal age at delivery time, infant birth weight and parity were included if significant at $\mathrm{p}<0.1{ }^{18}$ These covariates are considered by clinicians, and researchers working in the field as important and universally used in published papers on this topic. ${ }^{3-5}$ Multivariate binary logistic regression was conducted using the forced entry method. With respect to multivariate outliers and influential 
data points, all cases had Cook's D values below $1 .{ }^{19}$ No concerns regarding multicollinearity were noted and an acceptable goodness of fit model was observed. ${ }^{19}$

For mediation analysis data (BMI, vitamin D and depression) from 2013 to 2017 , ie 5 years $(n=8215$, no missing data) has been analysed. ${ }^{20}$ This is because, from 2013 onwards, maternal vitamin D status was measured and documented routinely by the hospital. The independent variable, early-pregnancy BMI, was treated as continuous, the mediator vitamin D status (deficient vs not deficient) and dependent variable, risk of perinatal depression (high vs low risk), were bi-categorical. Maternal early-pregnancy BMI was not normally distributed therefore the variable was log-transformed. However, log transformation did not alter the present findings, nor did it improve the distribution; thus, results are reported using original data. Baron and Kenny's (1986) guidelines were following to examine mediation (Figure 1); establish that the independent variable (early-pregnancy BMI) was associated with the mediator (vitamin D status) [Path a], and the mediator (vitamin D) was associated with the dependent variable (perinatal depression risk) [Path b] while simultaneously controlling for the independent variable (BMI) [Path c]. ${ }^{21}$ To test for mediation, unstandardized regression coefficients (B) and associated standard errors (SE) obtained from binary logistic regression were inserted into equations described by Iacobucci's (2012) ${ }^{22} \mathrm{Z}$ mediation

$$
=\frac{Z a Z b}{\sigma \mathrm{Zab}}=\frac{\mathrm{a}}{\mathrm{Sa}} * \frac{\frac{\mathrm{b}}{\mathrm{sb}}}{\sqrt{Z 2 a}}+z 2 b+1,
$$

Jose $(2013)^{23}$ and MacKinnon's (2008) ${ }^{24}$ SE

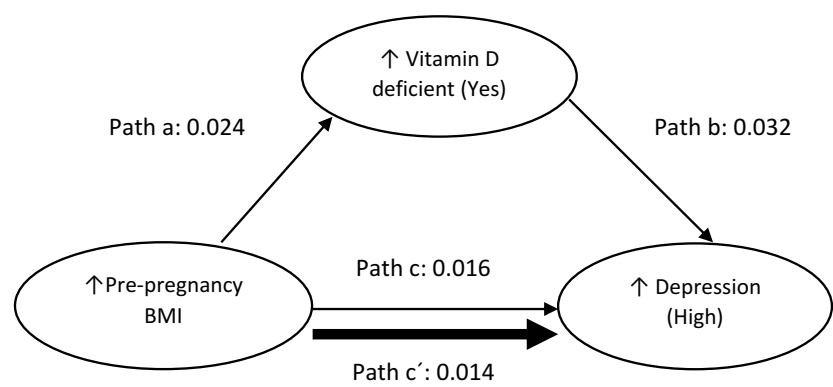

Figure I Mediational pathways between BMI, vitamin D status and perinatal depression risk. ${ }^{36,37}$

Note: Solid thick line indicates the association between BMI and perinatal depression risk to be partially mediated by vitamin D status. Data represent unstandardised regression coefficients.

Abbreviation: BMI, Body Mass Index.

$$
=\frac{a * b \mathrm{SQRT}\left[(\mathrm{t}-\text { score of } \mathrm{a})^{2}+(\mathrm{t}-\text { score of } \mathrm{b})^{2}\right]}{(\mathrm{t}-\text { score of } \mathrm{a}) *(\mathrm{t}-\text { score of } \mathrm{b})}
$$

and Sobel $(1982)^{25}$

$$
\mathrm{z}-\text { value }=\mathrm{a} * \mathrm{~b} / \operatorname{SQRT}\left(\mathrm{b}^{2} * \mathrm{~s}_{\mathrm{a}}^{2}+\mathrm{a}^{2} * \mathrm{~s}_{\mathrm{b}}^{2}\right) .
$$

Significant $p$ values $(p<0.05)$ indicated the presence of mediation and provided the proportion of Indirect Effect (IE) that was mediated. As the mediator (vitamin D) and dependent variable (depression) were bicategorical in nature the aforementioned equations were used to test for mediation as recommended by Hayes et al. ${ }^{26,27}$

\section{Results}

The majority of women in this cohort were born in Australia (67.2\%) and lived with a partner (89.1\%). Almost half of them worked (46.7\%) and only 9.0\% reported smoking during pregnancy. The distribution of births was similar during seasons, with $25.8 \%$ during Autumn, $25.1 \%$ during Winter, $32.1 \%$ in Summer and $17.0 \%$ in Spring (Table 1).

Adjusted logistic regression models reported that high maternal early-pregnancy BMI was associated with increased odds of having a greater risk of perinatal depression (AOR 1.421; 95\% CI, 1.191, 1.696: $\mathrm{p}<0.001$ ). High early-pregnancy BMI was associated with increased odds of being vitamin D deficient (AOR 1.950; 95\% CI; 1.735, $2.191, \mathrm{p}<0.001)$. In comparison to women with low perinatal depression risk, women with high perinatal depression risk had increased odds of being vitamin $\mathrm{D}$ deficient (AOR 1.321; 95\% CI, 1.105, 1.579: $\mathrm{p}=0.002$ ) (Table 2).

These results supported the conduction of mediational analysis. Sobel's test ${ }^{25}$ confirmed vitamin D deficiency significantly mediated the relationship between high earlypregnancy BMI and perinatal depression risk, test statistics $=3.1521, \mathrm{SE}=0.0025, \mathrm{p}=0.001$. Similarly, based on Jose (2013) ${ }^{23}$ and MacKinnon's (2008) ${ }^{24}$ equations there was a significant indirect effect of maternal earlypregnancy BMI on perinatal depression risk through vitamin D deficiency, IE (Indirect Effect) $=0.0024$ (95\% CI $0.0009,0.0038), z=3.1521, p=0.0016$. This was further confirmed based on Iacobucci's equation $(2012)^{22}$ which reported that there was a significant indirect effect of maternal early-pregnancy BMI on the risk of perinatal depression through vitamin D deficiency, IE $=22.2273$, $\mathrm{SE}=7.1221, \mathrm{z}=3.1209, \mathrm{p}=0.0018$. Based on MacKinnon $(2008)^{24}$ equations the proportion of total effect size that was partially mediated by vitamin $\mathrm{D}$ equalled 0.2350 ie 
Table I Descriptive Characteristics of the Obstetric Cohort

\begin{tabular}{|l|l|l|}
\hline Variables & $\begin{array}{l}\mathbf{n}(\%) \text { or } \\
\text { Mean } \pm \text { SD }\end{array}$ & $\begin{array}{l}\text { Minimum- } \\
\text { Maximum }\end{array}$ \\
\hline IV & & \\
BMI (n= 16,528) & $25.30 \pm 6.320$ & $13-77$ \\
Healthy weight 18.5-24.9 & $8338(50.4)$ & \\
Underweight <18.5 & $1012(6.1)$ & \\
Overweight 25-29.9 & $3904(23.6)$ & \\
Obese >30.0 & $3274(19.8)$ & \\
\hline DV & & \\
Vitamin D deficient (Yes) & $4792(50.0)$ & \\
Depression (High) & $948(6.7)$ & \\
\hline Covariates & & \\
Mum COB (Australia) & $11,077(67.2)$ & \\
Marital Status (With Partner) & $14,524(89.1)$ & \\
Mother Working (Yes) & $7718(46.7)$ & \\
Smokes during pregnancy (Yes) & $1486(9.0)$ & \\
Domestic Violence (Yes) & $86(0.5)$ & \\
Hypertension during pregnancy (Yes) & $838(5.1)$ & \\
Season of Birth (Summer) & $5310(32.1)$ & \\
Season of Birth (Autumn) & $4267(25.8)$ & \\
Season of Birth (Winter) & $4142(25.1)$ & \\
Season of Birth (Spring) & $2809(17.0)$ & \\
Gender (baby) (Female) & $8031(48.6)$ & \\
Maternal age at delivery time (years) & $31.06 \pm 5.176$ & \\
(n= I6,528) & 3331.84 & \\
Birth Weight (grams) (n= 16,528) & \pm 632.993 & \\
Parity (n= 16,528) & $0.90 \pm 1.08$ & $0-10$ \\
\hline
\end{tabular}

Abbreviations: IV, Independent Variable; DV, Dependent Variable; BMI, Body Mass Index; COB, Country Of Birth; SD, Standard Deviation.

approximately 24\%. Maternal early-pregnancy BMI was a weak significant predictor of perinatal depression risk after including vitamin $\mathrm{D}$ as a mediator, consistent with partial mediation, Path $\mathrm{C}: \mathrm{B}=0.016$ (95\% CI 1.003, 1.030), $\mathrm{p}=0.02$. Path $\mathrm{C}^{\prime}: \mathrm{B}=0.014$ (95\% CI 1.001, 1.028), $\mathrm{p}=0.04$ (Figure 1).

\section{Discussion}

As far as the authors are aware this is the first study to demonstrate that high maternal early-pregnancy BMI was no longer a significant predictor of perinatal depression risk after including the mediator (vitamin D) in the logistic regression model. Partial mediation was found with approximately $24 \%$ of the total effect of maternal obesity on low vitamin D status being explained by an increased risk of perinatal depression. The complexities associated with pregnancy-related depression need to be better understood to determine the mechanisms that contribute to its aetiology and the most effective interventions to prevent it.
Support for hypotheses one, that high maternal earlypregnancy BMI would be associated with an increased risk of perinatal depression, was found. Similar findings have been reported in the literature. Molyneaux et al. ${ }^{28}$ investigated the risk of antenatal and postpartum mental health disorders among obese and overweight women. In their systematic review and meta-analysis, 62 studies with a total of 540,373 women were included. Obese and overweight women had significantly higher odds of elevated depressive symptoms than normal-weight women. This was found during pregnancy (obese OR 1.43, 95\% CI 1.27-1.61, overweight OR 1.19, 95\% CI 1.09-1.31) and the postnatal period (obese OR 1.30, 95\% CI 1.20-1.42, overweight OR 1.09, 95\% CI 1.05-1.13). ${ }^{28}$

Our prediction, that high maternal early-pregnancy BMI would be associated with low vitamin D status, was also found to be correct. Bodnar et al. ${ }^{29}$ examined the relationship between maternal early-pregnancy BMI and 25hydroxyvitamin D [25(OH)D] concentrations. Serum 25 $(\mathrm{OH}) \mathrm{D}$ was measured at 4-21 weeks gestation in 400 pregnant women. Multivariable logistic regression models were used to assess the independent association between maternal early-pregnancy BMI and the odds of vitamin D deficiency $(<50 \mathrm{nmol} / \mathrm{L})$ after adjustment for ethnicity, season, gestational age, multivitamin use, physical activity, and maternal age. Compared with lean women (BMI $<25 \mathrm{~kg} / \mathrm{m} 2$ ), pregravid obese women $\left(\mathrm{BMI} \geq 30 \mathrm{~kg} / \mathrm{m}^{2}\right.$ ) had lower adjusted mean serum $25(\mathrm{OH})$ vitamin $\mathrm{D}$ concentrations at 4-22 weeks $(56.5$ verses $62.7 \mathrm{nmol} / \mathrm{L} ; \mathrm{P}<0.05)$ and a higher of prevalence vitamin D deficiency (61 versus $36 \%$; $\mathrm{P}<0.01$ ). ${ }^{29}$

Our study supports previous research demonstrating a significant association between low serum vitamin $\mathrm{D}$ and perinatal depression. ${ }^{30} \mathrm{~A}$ recent systematic review and meta-analysis investigated the association of vitamin D deficiency with pregnancy-related depression. Nine longitudinal studies with 8470 participants were included. The authors reported maternal 25(OH)D serum levels $<50$ nmol/1 were associated with 2.67 times (OR 3.67; 95\% CI $1.72-7.85)$ increased risk of postpartum depression compared to maternal $25(\mathrm{OH}) \mathrm{D}$ levels $\geq 50 \mathrm{nmol} / \mathrm{l}^{12}$

There are strengths and limitations to our study that should be discussed. The most significant strength, as far as we are aware, is that this is the first study of its kind to be published. Data were collected in the course of routine care by obstetric and midwifery staff and therefore at risk of potential recording bias. Nevertheless, the large sample size is a significant strength. Weight and height are taken, on average, at 12-14 weeks gestation before any real 
Table 2 Association Between Pre-Pregnancy BMI, Perinatal Depression Risk and Vitamin D Status

\begin{tabular}{|c|c|c|c|c|}
\hline Pre-Pregnancy BMI & Unadjusted OR (95\% Cl) & $P$ value & Adjusted OR (95\% Cl) & $P$ value \\
\hline \multicolumn{5}{|c|}{ Predicting Depression from BMI (Unadjusted $n=14,056$, Adjusted $n=13,805)^{a, b}$} \\
\hline Underweight & $1.922(1.506,2.452)$ & $<0.001$ & $1.544(1.194,1.997)$ & 0.001 \\
\hline Overweight & $1.254(\mathrm{I} .06 \mathrm{I}, \mathrm{I} .482)$ & 0.008 & $1.220(1.026,1.451)$ & 0.024 \\
\hline Obese & $1.553(1.311,1.839)$ & $<0.001$ & $1.421(1.191,1.696)$ & $<0.000$ \\
\hline \multicolumn{5}{|c|}{ Predicting Vitamin D from BMI (Unadjusted $n=9583$, Adjusted $n=9561)^{a, c}$} \\
\hline Underweight & $1.413(1.194,1.673)$ & $<0.001$ & $1.204(1.009,1.437)$ & 0.040 \\
\hline Overweight & $1.299(1.176,1.434)$ & $<0.001$ & $1.374(1.238,1.525)$ & $<0.000$ \\
\hline Obese & $1.479(1.325,1.650)$ & $<0.001$ & $1.950(1.735,2.191)$ & $<0.000$ \\
\hline \multicolumn{5}{|c|}{ Predicting Depression from Vitamin D (Unadjusted $n=8215$, Adjusted $n=8088)^{b, d}$} \\
\hline Vitamin D deficient (Yes) & $1.404(1.182,1.667)$ & $<0.001$ & $1.321(1.105,1.579)$ & 0.002 \\
\hline
\end{tabular}

Notes: Reference group: Healthy weight ${ }^{\mathrm{a}}$, Vitamin D deficient (No) ${ }^{\mathrm{d}, \mathrm{b}}$ Covariates Adjusted: Marital Status (With Partner), Mother Working (Yes), Smokes during pregnancy (Yes), Domestic Violence (Yes), Maternal age at delivery time (years), Birth weight, Parity. ${ }^{\mathrm{C}}$ Covariates Adjusted: Mum Country Of Birth (Australia), Maternal age at delivery time (years), gender, Birth weight, Parity.

Abbreviations: BMI, Body Mass Index; OR, Odds Ratio; Cl, Confidence Intervals.

impact of GWG is observed. We acknowledge, these values are still only an approximation of pre-pregnancy BMI. The 'first' documentation of pre-pregnancy weight at the study hospital is at the initial antenatal visit (typically around 14 weeks gestation) which is the routine practice throughout Australian maternity care institutions. A major limitation of the BOS software is that it does not allow the user to record actual BMI but is rounded up to the nearest whole figure instead. BMI has been used as a categorical variable in many similar studies investigating the association of maternal pre-pregnancy BMI and factors of interest. ${ }^{3-5}$ It should be noted that further stratification of weight may yield important health insights (eg, BMI 30-40, BMI 41-50, BMI 51-60 et.). However, we analysed all obesity classes together to preserve power. We found a ' $U$ ' shaped curve with underweight, overweight and obese women demonstrating a greater risk of both perinatal depression and vitamin $\mathrm{D}$ deficiency when compared to women of normal weight. This finding provided a rationale for the next step in our statistical analysis. The results of this study are clinically transferable by emphasising the need for risk reduction screening (and in this case vitamin D status and perinatal depression) in pregnant women who are outside of the 'normal' BMI range. Ideally, we would have liked to control for gestational age at the time of initial BMI recording, but this data is not routinely collected in our dataset. Around the world, the EPDS is the most common and acceptable screening tool for identifying depressive symptoms in the perinatal period, with a reported sensitivity of $68-86 \%$ and specificity of $78-96 \%{ }^{14}$ An Australian study used the EPDS among a sample of 4148 women reporting a sensitivity of $100 \%$ and specificity of $89 \%{ }^{31}$ However, limitations with using the EPDS are that it was only routinely administered at one point in time, some of the questions have been criticised for being ambiguous and there is the risk of potential scoring error. ${ }^{32}$ However, clinical practice guidelines for depression and related disorders during the perinatal period recommend universal, routine antenatal screening for depressive symptoms using the EPDS. ${ }^{33,34}$

Historically, vitamin D status has not been routinely tested for all pregnant women receiving antenatal care at the study hospital. More regular testing and documentation commenced in 2013; therefore, we can only include data from that year onwards. Serum levels are typically measured at the beginning of the second trimester (12-14 weeks). However, the exact timing of testing may differ for some women. In addition, we cannot exclude the possibility of residual confounding by factors known to influence vitamin D status such as maternal sun or Ultra Violet light exposure, physiological factors (such as dark skin pigmentation, certain medical disorders), vitamin/ mineral supplementation use and dietary intake of foods rich in this micronutrient. ${ }^{35}$ Lastly, our 5 -year cohort data are cross-sectional in nature. Therefore, we can confirm an association between BMI, vitamin D and depression but casual relationships cannot be ascertained.

The most common psychiatric disorders during pregnancy and the post-partum are depression and anxiety 
which are associated with high rates of both maternal and neonatal morbidity. The consequences of perinatal depression and anxiety can be both catastrophic and lifelong for both the mother and baby. ${ }^{13-15}$ Future research should include a more comprehensive examination of women's vitamin $\mathrm{D}$ status in association with sun exposure, eating behaviours and supplement use. This research should be combined with barriers that are commonly present in women who are overweight or obese, as these predictors increase the risk of developing perinatal depression.

\section{Conclusion}

In summary, we found that low levels of serum vitamin $\mathrm{D}$ in the second trimester of pregnancy are associated with a higher risk of developing perinatal depression. Earlypregnancy BMI was no longer a significant predictor of perinatal depression risk after including the mediator, vita$\min \mathrm{D}$, in the logistic regression model. In addition to the positive impacts on health and development of the offspring, ensuring adequate intake of vitamin D during pregnancy may be one method of reducing perinatal depression risk in women. Consistent with current recommendations, women with high early-pregnancy BMI should be screened for both depression risk and vitamin D insufficiency, as should women of all BMIs, with referral to relevant support services when indicated.

\section{Abbreviations}

BMI, Body Mass Index; EPDS, Edinburgh Postnatal Depression Scale; PPV, positive predictive value.

\section{Data Sharing Statement}

Data are available from the authors on reasonable request.

\section{Ethics and Consent Statement}

Our study was approved by the ACT Human Research Ethics Committee and the University of Canberra Human Research Ethics Committee (No:11.167). This study was classified as low risk by both ethics' committees named above. Data contained in the BOS were already deidentified by the hospital administration before researchers had access to it. At all times, this study complied with the ethical standards as set out by the Declaration of Helsinki.

\section{Author Contributions}

CKA, MB, RJ and MT made substantial contributions to conception and design plus data acquisition. RJ made substantial contributions to data analysis. CKA, MB, RJ and MT all contributed to drafting the article and then critically revising it for important intellectual content. All authors gave final approval of the version to be published and have an agreement to be accountable for all aspects of the work in ensuring that questions related to the accuracy or integrity of the work are appropriately investigated and resolved.

\section{Disclosure}

The authors have no conflict of interests regarding this work.

\section{References}

1. Chen $\mathrm{C}, \mathrm{Xu} \mathrm{X}$, Yan Y. Estimated global overweight and obesity burden in pregnant women based on panel data model. PLoS One. 2018;13:e0202183. doi:10.1371/journal.pone.0202183

2. Australian Bureau Statistics. The Australian health survey: first results 2011-12 BMI by age and sex. Australian government; 2012. Available from: http://www.rhwa.org.au. Accessed March 18, 2020.

3. Knight-Agarwal CR, Williams LT, Davis D, et al. Association of BMI and interpregnancy BMI change with birth outcomes in an Australian obstetric population: a retrospective cohort study. $B M J$ Open. 2016;6(5):e010667. doi:10.1136/bmjopen-2015-010667

4. Snowden JM, Mission JF, Marshall NE, et al. The impact of maternal obesity and race/ethnicity on perinatal outcomes: independent and joint effects. Obesity. 2016;24(7):1590-1598. doi:10.1002/oby.v24.7

5. Yang Z, Phung H, Freebairn L, Sexton R, Raulli A, Kelly P. Contribution of maternal overweight and obesity to the occurrence of adverse pregnancy outcomes. Aust N Z J Psychiatry. 2019;59 (3):367-374. doi:10.1111/ajo.2019.59.issue-3

6. Kumpulainen SM, Girchenko P, Lahti-Pulkkinen M, et al. Maternal early pregnancy obesity and depressive symptoms during and after pregnancy. Psychol Med. 2018;48(14):2353-2363. doi:10.1017/ S0033291717003889

7. Wortsman J, Matsuoka LY, Chen TC, Lu Z, Holick MF. Decreased bioavailability of vitamin D in obesity. Am J Clin Nutr. 2000;72 (3):690-693. doi:10.1093/ajen/72.3.690

8. Aghajafari F, Letourneau N, Mahinpey N, Cosic N, Giesbrecht G. Vitamin D deficiency and antenatal and postpartum depression: a systematic review. Nutrients. 2018;10(4):478. doi:10.3390/ nu10040478

9. Gale CR, Robinson SM, Harvey NC, et al. Maternal vitamin D status during pregnancy and child outcomes. Eur J Clin Nutr. 2008;62 (1):68. doi:10.1038/sj.ejen.1602680

10. Ginde AA, Sullivan AF, Mansbach JM, Camargo CA Jr. Vitamin $\mathrm{D}$ insufficiency in pregnant and nonpregnant women of childbearing age in the United States. Am J Obstet Gynecol. 2010;202(5):436-e1. doi:10.1016/j.ajog.2009.11.036

11. Holton S, Fisher J, Nguyen H, Brown WJ, Tran T. Pre-pregnancy body mass index and the risk of antenatal depression and anxiety. Women Birth. 2019;32(6):e508-e514. doi:10.1016/j.wombi.2019. 01.007

12. Wang J, Liu N, Sun W, Chen D, Zhao J, Zhang W. Association between vitamin $\mathrm{D}$ deficiency and antepartum and postpartum depression: a systematic review and meta-analysis of longitudinal studies. Arch Gynecol Obstet. 2018;298(6):1045-1059. doi:10.1007/ s00404-018-4902-6

13. Banti S, Mauri M, Oppo A, et al. From the third month of pregnancy to 1 year postpartum. prevalence, incidence, recurrence, and new onset of depression. results from the perinatal depression-research \& screening unit study. Compr Psychiatry. 2011;52(4):343-351. doi:10.1016/j.comppsych.2010.08.003 
14. Pearlstein T. Depression during pregnancy. Best Prac Res Clin Obstet Gynecol. 2015;29(5):754-764. doi:10.1016/j.bpobgyn.2015.04.004

15. Shakeel N, Eberhard-Gran M, Sletner L, et al. A prospective cohort study of depression in pregnancy, prevalence and risk factors in a multi-ethnic population. BMC Preg Childbirth. 2015;15(1):5. doi:10.1186/s12884-014-0420-0

16. Royal Australian New Zealand College Obstetrics Gynaecology (RANZCOG). Management of obesity in pregnancy statement C-Obs 49. RANZCOG; 2013. Available from: https://www.ranzcog. edu.au/RANZCOG_SITE/media. Accessed March 18, 2020.

17. Cox JL, Holden JM, Sagovsky R. Detection of postnatal depression: development of the 10-item Edinburgh postnatal depression scale. Brit J Psychiatry. 1987;150(6):782-786. doi:10.1192/bjp.150.6.782

18. Jani Mehta R, Mallan KM, Mihrshahi S, Mandalika S, Daniels LA. An exploratory study of associations between Australian-Indian mothers' use of controlling feeding practices, concerns and perceptions of children's weight and children's picky eating. Nutr Diet. 2014;71(1):28-34. doi:10.1111/ndi.2014.71.issue-1

19. Field A. Discovering Statistics Using IBM SPSS Statistics. Sage; 2013.

20. Preacher KJ, Hayes AF. SPSS and SAS procedures for estimating indirect effects in simple mediation models. Behav Res Methods, Instrum, Comput. 2004;36(4):717-731. doi:10.3758/BF03206553

21. Baron RM, Kenny DA. The moderator-mediator variable distinction in social psychological research: conceptual, strategic, and statistical considerations. J Pers Soc Psychol. 1986;51(6):1173. doi:10.1037/ 0022-3514.51.6.1173

22. Iacobucci D. Mediation analysis and categorical variables: the final frontier. J Consum Psychol. 2012;22(4):582-594. doi:10.1016/j. jcps.2012.03.006

23. Jose PE. Doing Statistical Mediation and Moderation. Guilford Press; 2013.

24. MacKinnon DP. Introduction to Statistical Mediation Analysis. New York: Taylor \& Francis; 2008

25. Sobel ME. Asymptotic confidence intervals for indirect effects in structural equation models. Sociol Methodol. 1982;13:290-312. doi: $10.2307 / 270723$

26. Hayes AF. PROCESS: a versatile computational tool for observed variable mediation, moderation, and conditional process modelling; 2012.

27. Hayes AF, Scharkow M. The relative trustworthiness of inferential tests of the indirect effect in statistical mediation analysis: does method really matter? Psychol Sci. 2013;24(10):1918-1927. doi:10.1177/095679 7613480187
28. Molyneaux E, Poston L, Ashurst-Williams S, Howard LM. Obesity and mental disorders during pregnancy and postpartum: a systematic review and meta-analysis. Obstet Gynecol. 2014;123(4):857. doi:10.1097/AOG.0000000000000170

29. Bodnar LM, Catov JM, Roberts JM, Simhan HN. Prepregnancy obesity predicts poor vitamin D status in mothers and their neonates. $J$ Nutr. 2007;137(11):2437-2442. doi:10.1093/jn/137. 11.2437

30. Robinson M, Whitehouse AJ, Newnham JP, et al. Low maternal serum vitamin $\mathrm{D}$ during pregnancy and the risk for postpartum depression symptoms. Arch Women's Men Health. 2014;17 (3):213-219. doi:10.1007/s00737-014-0422-y

31. Boyce P, Stubbs J, Todd A. The Edinburgh postnatal depression scale: validation for an Australian sample. Aust $N Z J$ Psychiatry. 1993;27(3):472-476. doi:10.3109/00048679309075805

32. Matthey S, Agostini F. Using the Edinburgh postnatal depression scale for women and men-some cautionary thoughts. Arch Women's Ment Health. 2017;20(2):345-354. doi:10.1007/s00737016-0710-9

33. Austin M-P, Highet N. Depression and related disorders - anxiety, bipolar disorder and puerperal psychosis - in the perinatal period. A Guideline for Primary Care Health Professionals Providing Care in the Perinatal Period. Melbourne: Beyondblue;2011. Available from: http://resources.beyondblue.org.au. Accessed March 18, 2020.

34. Venkatesh KK, Nadel H, Blewett D, et al. Implementation of universal screening for depression during pregnancy: feasibility and impact on obstetric care. Am J Obstet Gynecol. 2016;215(4):517. e1-517.e8. doi:10.1016/j.ajog.2016.05.024

35. Ogbo FA, Eastwood J, Hendry A, et al. Determinants of antenatal depression and postnatal depression in Australia. BMC Psychiatry. 2018;18(1):49. doi:10.1186/s12888-018-1598-x

36. Campbell KJ, Abbott G, Spence AC, Crawford DA, McNaughton SA, Ball K. Home food availability mediates associations between mothers' nutrition knowledge and child diet. Appetite. 2013;71:1-6. doi:10.1016/j.appet.2013.07.006

37. Lioret S, Cameron AJ, McNaughton SA, et al. Association between maternal education and diet of children at 9 months is partially explained by mothers' diet. Matern Child Nutr. 2015;11 (4):936-947. doi:10.1111/mcn.12031

\section{Publish your work in this journal}

The International Journal of Women's Health is an international, peerreviewed open-access journal publishing original research, reports, editorials, reviews and commentaries on all aspects of women's healthcare including gynecology, obstetrics, and breast cancer. The manuscript management system is completely online and includes a very quick and fair peer-review system, which is all easy to use. Visit http://www.dovepress.com/testimonials.php to read real quotes from published authors. 